\title{
OPTIMAL NETWORKS FOR MASS TRANSPORTATION PROBLEMS
}

\author{
Alessio Brancolini ${ }^{1}$ And Giuseppe Buttazzo ${ }^{2}$
}

\begin{abstract}
In the framework of transport theory, we are interested in the following optimization problem: given the distributions $\mu^{+}$of working people and $\mu^{-}$of their working places in an urban area, build a transportation network (such as a railway or an underground system) which minimizes a functional depending on the geometry of the network through a particular cost function. The functional is defined as the Wasserstein distance of $\mu^{+}$from $\mu^{-}$with respect to a metric which depends on the transportation network.
\end{abstract}

Mathematics Subject Classification. 49J45, 49Q10, 90B10.

Received October 21, 2003.

\section{INTRODUCTION}

Optimal Transportation Theory was first developed by Monge in 1781 in [12] where he raised the following question: given two mass distributions $f^{+}$and $f^{-}$, minimize the transport cost

$$
\int_{\mathbb{R}^{N}}|x-t(x)| f^{+}(x) \mathrm{d} x
$$

among all transport maps $t$, i.e. measurable maps such that the mass balance condition

$$
\int_{t^{-1}(B)} f^{+}(x) \mathrm{d} x=\int_{B} f^{-}(y) \mathrm{d} y
$$

holds for every Borel set $B$. Because of its strong non-linearity, Monge's formulation did not lead to significant advances up to 1940, when Kantorovich proposed his own formulation (see [10,11]).

In modern notation, given two finite positive Borel measures $\mu^{+}$and $\mu^{-}$on $\mathbb{R}^{N}$ such that $\mu^{+}\left(\mathbb{R}^{N}\right)=\mu^{-}\left(\mathbb{R}^{N}\right)$, Kantorovich was interested to minimize

$$
\int_{\mathbb{R}^{N} \times \mathbb{R}^{N}}|x-y| \mathrm{d} \mu(x, y)
$$

Keywords and phrases. Optimal networks, mass transportation problems.

1 Alessio Brancolini, Scuola Normale Superiore, Piazza dei Cavalieri 7, 56126 Pisa, Italy; e-mail: a.brancolini@sns.it

2 Giuseppe Buttazzo, Dipartimento di Matematica, Università di Pisa, Via Buonarroti 2, 56127 Pisa, Italy;

e-mail: buttazzo@dm.unipi.it 
among all transport plans $\mu$, i.e. positive Borel measures on $\mathbb{R}^{N} \times \mathbb{R}^{N}$ such that $\pi_{\#}^{+} \mu=\mu^{+}$and $\pi_{\#}^{-} \mu=\mu^{-}$, where by \# we denoted the push-forward operator $\left(\right.$ i.e. $\left.h_{\#} \mu(E)=\mu\left(h^{-1}(E)\right)\right)$. It is easy to see that if $t$ is a transport map between $\mu^{+}=f^{+} \mathcal{L}^{N}$ and $\mu^{-}=f^{-} \mathcal{L}^{N}$, then $(\operatorname{Id} \times t)_{\#} \mu^{+}$is a transport plan. So, Kantorovich's problem is a weak formulation of Monge's one.

Of course, one can take, instead of $\mathbb{R}^{N}$ and the cost function given by the Euclidean modulus, a generic pair of metric spaces $X$ and $Y$ and a positive lower semicontinuous cost function $c: X \times Y \rightarrow \mathbb{R}$, so that the Kantorovich problem reads:

$$
\min \left\{\int_{X \times Y} c(x, y) \mathrm{d} \mu(x, y): \pi_{\#}^{+} \mu=\mu^{+}, \pi_{\#}^{-} \mu=\mu^{-}\right\} .
$$

We stress the fact that $\mu^{+}$and $\mu^{-}$must have the same mass, otherwise there are no transport plans.

If we set $X=Y$ and take as cost function the distance $d$ in $X$, then the minimal value in (1.1) is called Wasserstein distance (of power 1) between $\mu^{+}$and $\mu^{-}$. In this case, we shall write $W_{d}\left(\mu^{+}, \mu^{-}\right)$.

For other details on transportation problems on networks we refer the interested reader to $[2,3,5-7,13]$.

\section{The OPTIMAL NETWORK PROBLEM}

We consider a bounded connected open subset $\Omega$ with Lipschitz boundary of $\mathbb{R}^{N}$ (the urban area) with $N>1$ and two positive finite measures $\mu^{+}$and $\mu^{-}$on $K:=\bar{\Omega}$ (the distributions of working people and of working places). We assume that $\mu^{+}$and $\mu^{-}$have the same mass that we normalize both equal 1 , that is $\mu^{+}$and $\mu^{-}$ are probability measures on $K$.

In this section we introduce the optimization problem for transportation networks: to every "urban network" $\Sigma$ we may associate a suitable "cost function" $d_{\Sigma}$ which takes into account the geometry of $\Sigma$ as well as the costs for customers to move with their own means and by means of the network. The cost functional will be then

$$
T(\Sigma)=W_{d_{\Sigma}}\left(\mu^{+}, \mu^{-}\right)
$$

so that the optimization problem we deal with is

$$
\min \{T(\Sigma): \Sigma \text { "admissible network" }\}
$$

The main result of this paper is to prove that, under suitable and very mild assumptions, and taking as admissible networks all connected, compact one-dimensional subsets $\Sigma$ of $K$, the optimization problem (2.2) admits a solution. The tools we use to obtain the existence result are a suitable relaxation procedure to define the function $d_{\Sigma}$ (Th. 4.2) and a generalization of the Gołab theorem (Th. 3.3), also obtained by Dal Maso and Toader in [8].

In order to introduce the distance $d_{\Sigma}$ we consider a function $J:[0,+\infty]^{3} \rightarrow[0,+\infty]$. For a given path $\gamma$ in $K$ the parameter $a$ in $J(a, b, c)$ measures the length of $\gamma$ outside $\Sigma, b$ measures the length of $\gamma$ inside $\Sigma$, while $c$ represents the total length of $\Sigma$. The $\operatorname{cost} J(a, b, c)$ is then the cost of a customer who travels for a length $a$ by his own means and for a length $b$ on the network, being $c$ the length of the latter. For instance we could take $J(a, b, c)=A(a)+B(b)+C(c)$ and then the function $A(t)$ is the cost for traveling a length $t$ by one's own means, $B(t)$ is the price of a ticket to cover the length $t$ on $\Sigma$ and $C(t)$ represents the cost of a network of length $t$.

For every closed connected subset $\Sigma$ in $K$, we define the cost function $d_{\Sigma}$ as

$$
d_{\Sigma}(x, y):=\inf \left\{J\left(\mathcal{H}^{1}(\gamma \backslash \Sigma), \mathcal{H}^{1}(\gamma \cap \Sigma), \mathcal{H}^{1}(\Sigma)\right): \gamma \in \mathscr{C}_{x, y}\right\}
$$

where $\mathscr{C}_{x, y}$ is the class of all closed connected subsets of $K$ containing $x$ and $y$. The optimization problem we consider is then (2.2) where we take as admissible networks all closed connected subsets $\Sigma$ of $K$ with 
$\mathcal{H}^{1}(\Sigma)<+\infty$. We also define, for every closed connected subset $\gamma$ of $K$

$$
L_{\Sigma}(\gamma):=J\left(\mathcal{H}^{1}(\gamma \backslash \Sigma), \mathcal{H}^{1}(\gamma \cap \Sigma), \mathcal{H}^{1}(\Sigma)\right) .
$$

We assume that $J$ satisfies the following conditions:

- $J$ is lower semicontinuous;

- $J$ is non-decreasing, i.e.

$$
a_{1} \leq a_{2}, b_{1} \leq b_{2}, c_{1} \leq c_{2} \Longrightarrow J\left(a_{1}, b_{1}, c_{1}\right) \leq J\left(a_{2}, b_{2}, c_{2}\right) ;
$$

- $J(a, b, c) \geq G(c)$ with $G(c) \rightarrow+\infty$ when $c \rightarrow+\infty$;

- $J$ is continuous in its first variable.

A curve joining two points $x, y \in K$ is an element of the set

$$
\mathscr{C}_{x, y}:=\{\gamma \text { closed connected, }\{x, y\} \subseteq \gamma \subseteq K\}
$$

while an element of $\mathscr{C}$ will be, by definition, a closed connected set in $K$ :

$$
\mathscr{C}:=\{\gamma \text { closed connected, } \gamma \subseteq K\} .
$$

We associate to every admissible network $\Sigma \in \mathscr{C}$ the cost function

$$
d_{\Sigma}(x, y)=\inf \left\{L_{\Sigma}(\gamma): \gamma \in \mathscr{C}_{x, y}\right\}
$$

We are interested in the functional $T$ given by

$$
\Sigma \mapsto T(\Sigma):=W_{d_{\Sigma}}\left(\mu^{+}, \mu^{-}\right)
$$

which is defined on the class $\mathscr{C}$, where the Wasserstein distance is defined in the introduction.

Finally by $\bar{L}_{\Sigma}^{x, y}$ we denote the lower semicontinuous envelope of $L_{\Sigma}$ with respect to the Hausdorff convergence on $\mathscr{C}_{x, y}$ (see Sect. 3 for the main definitions). In other words, for every $\gamma \in \mathscr{C}_{x, y}$ we set

$$
\bar{L}_{\Sigma}^{x, y}(\gamma)= \begin{cases}\min \left\{\liminf _{n} L_{\Sigma}\left(\gamma_{n}\right): \gamma_{n} \rightarrow \gamma, \gamma_{n} \in \mathscr{C}_{x, y}\right\} & \text { if } \gamma \in \mathscr{C}_{x, y} \\ +\infty & \text { if } \gamma \notin \mathscr{C}_{x, y}\end{cases}
$$

where we fix the condition $x, y \in \gamma$. Moreover, we define $\bar{L}_{\Sigma}$ as

$$
\bar{L}_{\Sigma}(\gamma)=\min \left\{\liminf _{n \rightarrow+\infty} L_{\Sigma}\left(\gamma_{n}\right): \gamma_{n} \rightarrow \gamma, \gamma_{n} \in \mathscr{C}\right\}
$$

that is to say, the lower semicontinuous envelope of $L_{\Sigma}$ with respect to the Hausdorff convergence on the class of closed connected sets of $K$.

\section{The Golab theorem And its extensions}

In this section $X$ will be a set endowed with a distance function $d$, i.e. $(X, d)$ is a metric space. We assume for simplicity $X$ to be compact. By $\mathscr{C}(X)$ we indicate the class of all closed subsets of $X$.

Given two closed subsets $C$ and $D$, the Hausdorff distance between them is defined by

$$
d_{\mathcal{H}}(C, D):=1 \wedge \inf \left\{r \in \left[0,+\infty\left[: C \subseteq D_{r}, D \subseteq C_{r}\right\}\right.\right.
$$


where

$$
C_{r}:=\{x \in X: d(x, C)<r\}
$$

It is easy to see that $d_{\mathcal{H}}$ is a distance on $\mathscr{C}(X)$, so $\left(\mathscr{C}(X), d_{\mathcal{H}}\right)$ is a metric space. We remark the following well-known facts (see for example [1]):

- $(X, d)$ compact $\Longrightarrow\left(\mathscr{C}(X), d_{\mathcal{H}}\right)$ compact;

- $(X, d)$ complete $\Longrightarrow\left(\mathscr{C}(X), d_{\mathcal{H}}\right)$ complete.

In the rest of the paper we will use the notation $C_{n} \rightarrow C$ to indicate the convergence of a sequence $\left\{C_{n}\right\}_{n \in \mathbb{N}}$ to $C$ with respect to the distance $d_{\mathcal{H}}$.

Proposition 3.1. Let $\left\{C_{n}\right\}_{n \in \mathbb{N}}$ be a sequence of compact connected subsets in $X$ such that $C_{n} \rightarrow C$ for some compact subset $C$. Then $C$ is connected.

Proof. Suppose, on the contrary, that there exist two closed non-void separated subsets $F_{1}$ and $F_{2}$ such that $C=F_{1} \cup F_{2}$. Since $F_{1}$ and $F_{2}$ are compact, $d\left(F_{1}, F_{2}\right)=d>0$. Let us choose $\varepsilon=d / 4$. By the definition of Hausdorff convergence, there exists a positive integer $N$ such that

$$
n \geq N \Longrightarrow C_{n} \subseteq(C)_{\varepsilon}, C \subseteq\left(C_{n}\right)_{\varepsilon}
$$

Since $C_{N}$ is connected, we must have either $C_{N} \subseteq\left(F_{1}\right)_{\varepsilon}$ or $C_{N} \subseteq\left(F_{2}\right)_{\varepsilon}$. Let us suppose, for example, that $C_{N} \subseteq\left(F_{1}\right)_{\varepsilon}$. On one side by the Hausdorff convergence it is $F_{2} \subseteq\left(C_{N}\right)_{\varepsilon}$, on the other by the choice of $\varepsilon$ we have $\left(C_{N}\right)_{\varepsilon} \cap F_{2}=\varnothing$, a contradiction.

The Hausdorff 1-dimensional measure in $(X, d)$ of a Borel set $B$ is defined by

$$
\mathcal{H}^{1}(B):=\lim _{\delta \rightarrow 0^{+}} \mathcal{H}^{1, \delta}(B)
$$

where

$$
\mathcal{H}^{1, \delta}(B):=\inf \left\{\sum_{n \in \mathbb{N}} \operatorname{diam} B_{n}: \operatorname{diam} B_{n}<\delta, B \subseteq \bigcup_{n \in \mathbb{N}} B_{i}\right\} .
$$

The measure $\mathcal{H}^{1}$ is Borel regular and if $(X, d)$ is the 1 -dimensional Euclidean space, then $\mathcal{H}^{1}$ is just the Lebesgue measure $\mathcal{L}^{1}$

The Golab classical theorem states that in a metric space, the measure $\mathcal{H}^{1}$ is sequentially lower semicontinuous with respect to the Hausdorff convergence over the class of all compact connected subsets of $X$.

Theorem 3.2 (Gołab). Let $X$ be a metric space. If $\left\{C_{n}\right\}_{n \in \mathbb{N}}$ is a sequence of compact connected subsets of $X$ and $C_{n} \rightarrow C$ for some compact connected subset $C$, then

$$
\mathcal{H}^{1}(C) \leq \liminf _{n \rightarrow+\infty} \mathcal{H}^{1}\left(C_{n}\right)
$$

Actually, this result can be strengthened.

Theorem 3.3. Let $X$ be a metric space, $\left\{\Gamma_{n}\right\}_{n \in \mathbb{N}}$ and $\left\{\Sigma_{n}\right\}_{n \in \mathbb{N}}$ be two sequences of compact subsets such that $\Gamma_{n} \rightarrow \Gamma$ and $\Sigma_{n} \rightarrow \Sigma$ for some compact subsets $\Gamma$ and $\Sigma$. Let us also suppose that $\Gamma_{n}$ is connected for all $n \in \mathbb{N}$. Then

$$
\mathcal{H}^{1}(\Gamma \backslash \Sigma) \leq \liminf _{n \rightarrow+\infty} \mathcal{H}^{1}\left(\Gamma_{n} \backslash \Sigma_{n}\right) .
$$

A proof of this result has been given by Dal Maso and Toader in [8]; for sake of completeness, we include the proof here below. It is in fact based on the following two rectifiability theorems whose proof can be found in [1].

Theorem 3.4. Let $X$ be a metric space and $C$ a closed connected subset of finite length, i.e. $\mathcal{H}^{1}(C)<+\infty$. Then $C$ is compact and connected by injective rectifiable curves. 
Theorem 3.5. Let $C$ be a closed connected subset in a metric space $X$ such that $\mathcal{H}^{1}(C)<+\infty$. Then there exists a sequence of Lipschitz curves $\left\{\gamma_{n}\right\}_{n \in \mathbb{N}}, \gamma_{n}:[0,1] \rightarrow C$, such that

$$
\mathcal{H}^{1}\left(C \backslash \bigcup_{n \in \mathbb{N}} \gamma_{n}([0,1])\right)=0 .
$$

The first step in the proof of Theorem 3.3 is a localized form of the Gołab classical theorem. To this aim we need the following lemma.

Lemma 3.6. Let $C$ be a closed connected subset of $X$ and let $x \in C$. If $r \in\left[0, \frac{1}{2}\right.$ diam $\left.C\right]$, then

$$
\mathcal{H}^{1}\left(C \cap B_{r}(x)\right) \geq r
$$

Proof. See for instance Lemma 4.4.2 of [1] or Lemma 3.4 of [9].

Remark 3.7. Lemma 3.6 yields the following estimate from below for the upper density:

$$
\bar{\theta}(C, x):=\limsup _{r \rightarrow 0^{+}} \frac{\mathcal{H}^{1}\left(C \cap B_{r}(x)\right)}{2 r} \geq \frac{1}{2} .
$$

We recall that for every measure $\mu$ the upper density is defined by

$$
\bar{\theta}(\mu, x):=\limsup _{r \rightarrow 0^{+}} \frac{\mu\left(B_{r}(x)\right)}{2 r} .
$$

We also recall that $\bar{\theta}(\mu, x) \geq t$ for all $x \in X$ implies $\mu(B) \geq t \mathcal{H}^{1}(B)$ for every Borel set $B$ (see Th. 2.4.1 in [1]).

We are now in a position to obtain the localized version of the Gołab theorem.

Theorem 3.8. Let $X$ be a metric space. If $\left\{C_{n}\right\}_{n \in \mathbb{N}}$ is a sequence of compact connected subsets of $X$ such that $C_{n} \rightarrow C$ for some compact connected subset $C$, then for every open subset $U$ of $X$

$$
\mathcal{H}^{1}(C \cap U) \leq \liminf _{n \rightarrow+\infty} \mathcal{H}^{1}\left(C_{n} \cap U\right) .
$$

Proof. We can suppose that $L:=\lim _{n} \mathcal{H}^{1}\left(C_{n} \cap U\right)$ exists, is finite and $\mathcal{H}^{1}\left(C_{n} \cap U\right) \leq L+1$. Let $d_{n}=$ $\operatorname{diam}\left(C_{n} \cap U\right)$. We can suppose up to a subsequence that $d_{n} \rightarrow d>0$. Let us consider the sequence of Borel measures defined by

$$
\mu_{n}(B):=\mathcal{H}^{1}\left(B \cap C_{n} \cap U\right)
$$

for every Borel set $B$. Up to a subsequence we can assume that $\mu_{n} \rightarrow^{*} \mu$ for a suitable $\mu$. We choose $x \in C \cap U$ and $r^{\prime}<r<\operatorname{diam}(C \cap U) / 2$. Then, by Lemma 3.6,

$$
\mu\left(B_{r}(x)\right) \geq \mu\left(\bar{B}_{r^{\prime}}(x)\right) \geq \limsup _{n \rightarrow+\infty} \mu_{n}\left(\bar{B}_{r^{\prime}}(x)\right)=\limsup _{n \rightarrow+\infty} \mathcal{H}^{1}\left(C_{n} \cap \bar{B}_{r^{\prime}}(x) \cap U\right) \geq r^{\prime}
$$

Since $r^{\prime}$ was chosen arbitrarily we get

$$
\mu\left(B_{r}(x)\right) \geq r
$$

for every $x \in C \cap U$ and $r<\operatorname{diam}(C \cap U) / 2$. This implies $\bar{\theta}(C, x) \geq 1 / 2$. By Remark 3.7

$$
\mathcal{H}^{1}(C \cap U) \leq 2 \mu(X) \leq 2 \liminf _{n \rightarrow+\infty} \mu_{n}(X)=2 \liminf _{n \rightarrow+\infty} \mathcal{H}^{1}\left(C_{n} \cap U\right)=2 L
$$


By Theorem 3.5 for $\mathcal{H}^{1}$-almost all $x_{0} \in C \cap U$ there exists a Lipschitz curve $\gamma$ whose range is in $C \cap U$ such that $x_{0}=\gamma\left(t_{0}\right)$ and $\left.t_{0} \in\right] 0,1[$. We can also suppose that

$$
\lim _{h \rightarrow 0^{+}} \frac{d\left(\gamma\left(t_{0}+h\right), \gamma\left(t_{0}-h\right)\right)}{2|h|}=1 .
$$

We choose arbitrarily $\sigma \in] 0,1[$. If $h$ is small, then

$$
d\left(\gamma\left(t_{0}+h\right), \gamma\left(t_{0}-h\right)\right) \geq(2-\sigma)|h|
$$

and

$$
(1-\sigma)|h| \leq d\left(\gamma\left(t_{0} \pm h\right), \gamma\left(t_{0}\right)\right) \leq(1+\sigma)|h| .
$$

Let us also suppose that $|h|<\sigma /(1+\sigma)$ and put

$$
y:=\gamma\left(t_{0}-h\right), \quad z:=\gamma\left(t_{0}+h\right), \quad r:=\max \left\{d\left(y, x_{0}\right), d\left(z, x_{0}\right)\right\}
$$

We get

$$
r<(1+\sigma)|h|<\sigma, \quad d(y, z) \geq(2-\sigma)|h| \geq \frac{2-\sigma}{2+\sigma} r .
$$

Let $r^{\prime}:=(1+\sigma) r$. Since $C_{n} \rightarrow C$, then (see Prop. 4.4.3 in [1]) there exist subsequences $\left\{y_{n}\right\}_{n \in \mathbb{N}}$ and $\left\{z_{n}\right\}_{n \in \mathbb{N}}$ such that $y_{n}, z_{n} \in C_{n} \cap U, y_{n} \rightarrow y$ and $z_{n} \rightarrow z$. One must have $y_{n}, z_{n} \in B_{r^{\prime}}\left(x_{0}\right)$ for $n$ large enough and

$$
\mu_{n}\left(\overline{B_{r^{\prime}}(x)}\right)=\mathcal{H}^{1}\left(C_{n} \cap \overline{B_{r^{\prime}}(x)} \cap U\right) \geq d\left(z, y_{n}\right)
$$

Taking the limsup

$$
\begin{aligned}
\mu\left(\overline{B_{r^{\prime}}(x)}\right) & \geq \limsup _{n \rightarrow+\infty} \mathcal{H}^{1}\left(C_{n} \cap \overline{B_{r^{\prime}}(x)} \cap U\right) \geq \limsup _{n \rightarrow+\infty} d\left(z, y_{n}\right) \\
& =d(z, y) \geq \frac{2-\sigma}{2+\sigma} r=\frac{2-\sigma}{(2+\sigma)(1+\sigma)} r^{\prime}
\end{aligned}
$$

Since $\sigma$ was arbitrary, we get $\bar{\theta}\left(\mu, x_{0}\right) \geq 1$ for $\mathcal{H}^{1}$-almost all $x_{0} \in C \cap U$. Then, by Remark 3.7

$$
\mathcal{H}^{1}(C \cap U) \leq \mu(X) \leq \liminf _{n \rightarrow+\infty} \mu_{n}(X)=\liminf _{n \rightarrow+\infty} \mathcal{H}^{1}\left(C_{n} \cap U\right)
$$

Proof of Theorem 3.3. Let $A=\Gamma \cap \Sigma$. Thanks to the equality

$$
\bigcup_{\varepsilon>0}\left(\Gamma \backslash \bar{A}_{\varepsilon}\right)=\Gamma \backslash \Sigma
$$

we have

$$
\lim _{\varepsilon \rightarrow 0^{+}} \mathcal{H}^{1}\left(\Gamma \backslash \bar{A}_{\varepsilon}\right)=\mathcal{H}^{1}(\Gamma \backslash \Sigma)
$$

Recalling that the following inclusion of sets holds for large values of $n$

$$
\Gamma_{n} \backslash \bar{A}_{\varepsilon} \subseteq \Gamma_{n} \backslash A_{n} \subseteq \Gamma_{n} \backslash \Sigma_{n}
$$

by the localized form of Gołab theorem (Th. 3.8) we deduce

$$
\mathcal{H}^{1}\left(\Gamma \backslash \bar{A}_{\varepsilon}\right) \leq \liminf _{n \rightarrow+\infty} \mathcal{H}^{1}\left(\Gamma_{n} \backslash \bar{A}_{\varepsilon}\right) \leq \liminf _{n \rightarrow+\infty} \mathcal{H}^{1}\left(\Gamma_{n} \backslash \Sigma_{n}\right) .
$$


Taking the limit as $\varepsilon \rightarrow 0^{+}$, we obtain

$$
\mathcal{H}^{1}(\Gamma \backslash \Sigma) \leq \liminf _{n \rightarrow+\infty} \mathcal{H}^{1}\left(\Gamma_{n} \backslash \Sigma_{n}\right) .
$$

Remark 3.9. It is easy to see that if the number of connected components of $C_{n}$ is bounded from above by a positive integer independent on $n$, then the localized form of Gołab theorem is still valid. All details can be found in [8].

\section{Relaxation of the Cost FunCtion}

We can give an explicit expression for the lower semicontinuous envelopes $\bar{L}_{\Sigma}$ and $\bar{L}_{\Sigma}^{x, y}$ in terms of $J$. In order to achieve this result it is useful to introduce the function:

$$
\bar{J}(a, b, c)=\inf \{J(a+t, b-t, c): 0 \leq t \leq b\} .
$$

The following lemma is an important step to establish Theorem 4.2.

Lemma 4.1. Let $\gamma$ and $\Sigma$ be closed connected subsets of $K$. Let also suppose that $\Sigma$ has a finite length. Then for every $t \in\left[0, \mathcal{H}^{1}(\gamma \cap \Sigma)\right]$ we can find a sequence $\left\{\gamma_{n}\right\}_{n \in \mathbb{N}}$ in $\mathscr{C}$ such that

- $\gamma_{n} \rightarrow \gamma$

- $\lim _{n} \mathcal{H}^{1}\left(\gamma_{n}\right)=\mathcal{H}^{1}(\gamma)$

- $\mathcal{H}^{1}\left(\gamma_{n} \cap \Sigma\right) \nearrow \mathcal{H}^{1}(\gamma \cap \Sigma)-t$.

Moreover, if $x, y \in \gamma$ then the sequence $\left\{\gamma_{n}\right\}_{n \in \mathbb{N}}$ can be chosen in $\mathscr{C}_{x, y}$.

Proof. The set $\gamma \cap \Sigma$ is closed and with a finite length. By the second rectifiability result (Th. 3.5) it follows the existence of a sequence of curves $\sigma_{n} \in \operatorname{Lip}([0,1], K)$ such that

$$
\mathcal{H}^{1}\left((\gamma \cap \Sigma) \backslash \bigcup_{n \in \mathbb{N}} \sigma_{n}([0,1])\right)=0 .
$$

We can also suppose that the subsets $\sigma_{n}([0,1])$ are disjoint up to subsets of negligible length. Fix a sufficiently small $\delta>0$ and choose a sequence of intervals $I_{n}=\left[a_{n}, b_{n}\right]$ such that

$$
\sum_{n \in \mathbb{N}} \mathcal{H}^{1}\left(\sigma_{n}\left(I_{n}\right)\right)=t+\delta .
$$

For every sequence $\underline{v}=\left\{v_{n}\right\}_{n \in \mathbb{N}}$ of unit vectors of $\mathbb{R}^{N}$ such that $v_{n}$ is not tangent to $\gamma \cap \Sigma$ in $\sigma_{n}\left(a_{n}\right)$ and $\sigma_{n}\left(b_{n}\right)$, and every sequence $\underline{\varepsilon}=\left\{\varepsilon_{n}\right\}_{n \in \mathbb{N}}$ of positive real numbers, let us consider

$$
\begin{aligned}
A_{\underline{v}, \underline{\varepsilon}} & =\bigcup_{n \in \mathbb{N}} \sigma_{n}\left(\left[0, a_{n}\right] \cup\left[b_{n}, 1\right]\right), \\
B_{\underline{v}, \underline{\varepsilon}} & =\bigcup_{n \in \mathbb{N}}\left(\sigma_{n}\left(a_{n}\right)+\varepsilon_{n} V_{n}\right), \\
C_{\underline{v}, \underline{\varepsilon}} & =\bigcup_{n \in \mathbb{N}}\left(v_{n}+\sigma_{n}\left(I_{n}\right)\right), \\
D_{\underline{v}, \underline{\varepsilon}} & =\bigcup_{n \in \mathbb{N}}\left(\sigma_{n}\left(b_{n}\right)+\varepsilon_{n} V_{n}\right) \\
\gamma_{\underline{v}, \underline{\varepsilon}} & =(\gamma \backslash \Sigma) \cup A_{\underline{v}, \underline{\varepsilon}} \cup B_{\underline{v}, \underline{\varepsilon}} \cup C_{\underline{v}, \underline{\varepsilon}} \cup D_{\underline{v}, \underline{\varepsilon}}
\end{aligned}
$$

where $V_{n}=\left\{t v_{n}: t \in[0,1]\right\}$ (see Fig. 1). 


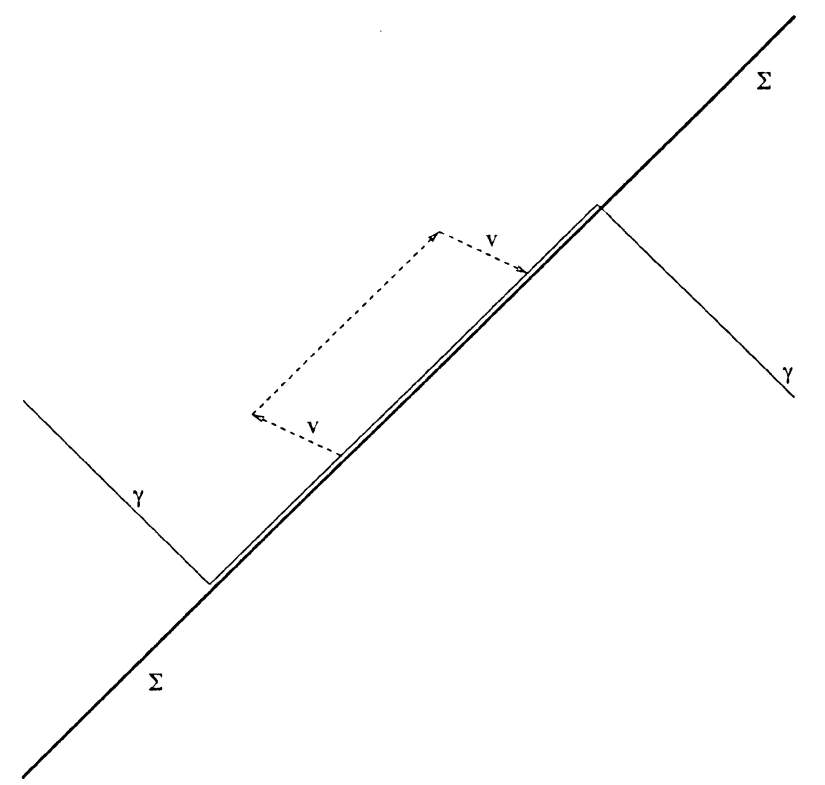

FiguRE 1. The approximating curves $\gamma_{n}$.

Since $\Sigma$ is closed and with a finite length, the class of $\gamma_{\underline{v}, \underline{\varepsilon}}$ that have not $\mathcal{H}^{1}$-negligible intersection with $\Sigma$ is at most countable. Out of that set we can choose sequences $\delta_{m} \searrow 0$, and $\left\{\gamma_{\underline{v}_{m}, \underline{\varepsilon}_{m}}\right\}_{m \in \mathbb{N}}$ such that $\left\|\underline{\varepsilon}_{m}\right\| \searrow 0$, where by $\|\underline{\varepsilon}\|$ we denote the quantity $\sum_{n} \varepsilon_{n}$. The sequence $\left\{\gamma_{\underline{v}_{m}, \underline{\varepsilon}_{m}}\right\}_{m \in \mathbb{N}}$ is the one we were looking for.

Theorem 4.2. For every closed connected subset $\gamma \in \mathscr{C}_{x, y}$ we have

$$
\bar{L}_{\Sigma}^{x, y}(\gamma)=\bar{J}\left(\mathcal{H}^{1}(\gamma \backslash \Sigma), \mathcal{H}^{1}(\gamma \cap \Sigma), \mathcal{H}^{1}(\Sigma)\right)
$$

Moreover, if $\gamma \in \mathscr{C}_{x, y}$ then

$$
\bar{L}_{\Sigma}^{x, y}(\gamma)=\bar{L}_{\Sigma}(\gamma)
$$

Proof. Let $\gamma$ be a fixed curve in $\mathscr{C}_{x, y}$. First we establish that

$$
\bar{L}_{\Sigma}^{x, y}(\gamma) \geq \bar{J}\left(\mathcal{H}^{1}(\gamma \backslash \Sigma), \mathcal{H}^{1}(\gamma \cap \Sigma), \mathcal{H}^{1}(\Sigma)\right)
$$

It is enough to show that for every sequence $\left\{\gamma_{n}\right\}_{n \in \mathbb{N}}$ in $\mathscr{C}_{x, y}$ converging to $\gamma$ with respect to the Hausdorff metric, there exists $t \in\left[0, \mathcal{H}^{1}(\gamma \cap \Sigma)\right]$ such that

$$
J\left(\mathcal{H}^{1}(\gamma \backslash \Sigma)+t, \mathcal{H}^{1}(\gamma \cap \Sigma)-t, \mathcal{H}^{1}(\Sigma)\right) \leq \liminf _{n \rightarrow+\infty} L_{\Sigma}\left(\gamma_{n}\right)
$$

Up to a subsequence we can suppose the following equalities hold true:

$$
\begin{aligned}
\liminf _{n \rightarrow+\infty} L_{\Sigma}\left(\gamma_{n}\right) & =\lim _{n \rightarrow+\infty} L_{\Sigma}\left(\gamma_{n}\right), \\
\liminf _{n \rightarrow+\infty} \mathcal{H}^{1}\left(\gamma_{n}\right) & =\lim _{n \rightarrow+\infty} \mathcal{H}^{1}\left(\gamma_{n}\right), \\
\liminf _{n \rightarrow+\infty} \mathcal{H}^{1}\left(\gamma_{n} \backslash \Sigma\right) & =\lim _{n \rightarrow+\infty} \mathcal{H}^{1}\left(\gamma_{n} \backslash \Sigma\right) .
\end{aligned}
$$


Moreover, by Gołab theorems (Ths. 3.2 and 3.3)

$$
\begin{aligned}
\mathcal{H}^{1}(\gamma) & \leq \lim _{n \rightarrow+\infty} \mathcal{H}^{1}\left(\gamma_{n}\right), \\
\mathcal{H}^{1}(\gamma \backslash \Sigma) & \leq \lim _{n \rightarrow+\infty} \mathcal{H}^{1}\left(\gamma_{n} \backslash \Sigma\right) .
\end{aligned}
$$

Choose $t=\lim _{n} \mathcal{H}^{1}\left(\gamma_{n} \backslash \Sigma\right)-\mathcal{H}^{1}(\gamma \backslash \Sigma)$. Then $\mathcal{H}^{1}(\gamma \backslash \Sigma)+t=\lim _{n} \mathcal{H}^{1}\left(\gamma_{n} \backslash \Sigma\right)$. We have

$$
\begin{aligned}
\mathcal{H}^{1}\left(\gamma_{n}\right) & =\mathcal{H}^{1}\left(\gamma_{n} \backslash \Sigma\right)+\mathcal{H}^{1}\left(\gamma_{n} \cap \Sigma\right) \\
& =\left[\mathcal{H}^{1}\left(\gamma_{n} \backslash \Sigma\right)-t\right]+\left[\mathcal{H}^{1}\left(\gamma_{n} \cap \Sigma\right)+t\right]
\end{aligned}
$$

Taking the limit as $n \rightarrow+\infty$ gives

$$
\mathcal{H}^{1}(\gamma) \leq \lim _{n \rightarrow+\infty} \mathcal{H}^{1}\left(\gamma_{n}\right)=\left[\mathcal{H}^{1}(\gamma \backslash \Sigma)+t\right]+\lim _{n \rightarrow+\infty} \mathcal{H}^{1}\left(\gamma_{n} \cap \Sigma\right)
$$

so that

$$
\mathcal{H}^{1}(\gamma \cap \Sigma)-t \leq \lim _{n \rightarrow+\infty} \mathcal{H}^{1}\left(\gamma_{n} \cap \Sigma\right)
$$

It follows by the semicontinuity and monotonicity of $J$ in the first two variables

$$
J\left(\mathcal{H}^{1}(\gamma \backslash \Sigma)+t, \mathcal{H}^{1}(\gamma \cap \Sigma)-t, \mathcal{H}^{1}(\Sigma)\right) \leq \liminf _{n \rightarrow+\infty} J\left(\mathcal{H}^{1}\left(\gamma_{n} \backslash \Sigma\right), \mathcal{H}^{1}\left(\gamma_{n} \cap \Sigma\right), \mathcal{H}^{1}(\Sigma)\right)
$$

Now, we have to establish the opposite inequality:

$$
\bar{L}_{\Sigma}^{x, y}(\gamma) \leq \bar{J}\left(\mathcal{H}^{1}(\gamma \backslash \Sigma), \mathcal{H}^{1}(\gamma \cap \Sigma), \mathcal{H}^{1}(\Sigma)\right)
$$

In the same way as before, it is enough to show that for every $t \in\left[0, \mathcal{H}^{1}(\gamma \cap \Sigma)\right]$ we can find a sequence $\left\{\gamma_{n}\right\}_{n \in \mathbb{N}}$ in $\mathscr{C}_{x, y}$ which converges to $\gamma$ such that

$$
\liminf _{n \rightarrow+\infty} L_{\Sigma}\left(\gamma_{n}\right) \leq J\left(\mathcal{H}^{1}(\gamma \backslash \Sigma)+t, \mathcal{H}^{1}(\gamma \cap \Sigma)-t, \mathcal{H}^{1}(\Sigma)\right)
$$

Given $t$, let $\left\{\gamma_{n}\right\}_{n \in \mathbb{N}}$ be the sequence given by Lemma 4.1. Then we get

$$
\lim _{n \rightarrow+\infty} \mathcal{H}^{1}\left(\gamma_{n} \backslash \Sigma\right)=\mathcal{H}^{1}(\gamma)-\mathcal{H}^{1}(\gamma \cap \Sigma)+t=\mathcal{H}^{1}(\gamma \backslash \Sigma)+t
$$

Thanks to $\mathcal{H}^{1}\left(\gamma_{n} \cap \Sigma\right) \leq \mathcal{H}^{1}(\gamma \cap \Sigma)-t$, we have

$$
J\left(\mathcal{H}^{1}\left(\gamma_{n} \backslash \Sigma\right), \mathcal{H}^{1}\left(\gamma_{n} \cap \Sigma\right), \mathcal{H}^{1}(\Sigma)\right) \leq J\left(\mathcal{H}^{1}\left(\gamma_{n} \backslash \Sigma\right), \mathcal{H}^{1}(\gamma \cap \Sigma)-t, \mathcal{H}^{1}(\Sigma)\right)
$$

and by the continuity of $J$ in the first variable

$$
\liminf _{n \rightarrow+\infty} J\left(\mathcal{H}^{1}\left(\gamma_{n} \backslash \Sigma\right), \mathcal{H}^{1}\left(\gamma_{n} \cap \Sigma\right), \mathcal{H}^{1}(\Sigma)\right) \leq J\left(\mathcal{H}^{1}(\gamma \backslash \Sigma)+t, \mathcal{H}^{1}(\gamma \cap \Sigma)-t, \mathcal{H}^{1}(\Sigma)\right)
$$

which implies the inequality we looked for. The proof of the second statement of the theorem is analogous and hence omitted.

The next proposition is a consequence of Theorem 4.2.

Proposition 4.3. For every $x, y \in K$ we have

$$
d_{\Sigma}(x, y)=\inf \left\{\bar{L}_{\Sigma}(\gamma): \gamma \in \mathscr{C}_{x, y}\right\}
$$


Proof. By a general result of relaxation theory (see for instance [4]), the infimum of a function is the same as the infimum of its lower semicontinuous envelope, so

$$
d_{\Sigma}(x, y)=\inf \left\{\bar{L}_{\Sigma}^{x, y}(\gamma): \gamma \in \mathscr{C}_{x, y}\right\}
$$

It is then enough to prove that

$$
\inf \left\{\bar{L}_{\Sigma}^{x, y}(\gamma): \gamma \in \mathscr{C}_{x, y}\right\}=\inf \left\{\bar{L}_{\Sigma}(\gamma): \gamma \in \mathscr{C}_{x, y}\right\}
$$

which is a consequence of Theorem 4.2.

It is more convenient to introduce the function whose variables $a, b, c$ now represent the length $\mathcal{H}^{1}(\gamma \backslash \Sigma)$ covered by one's own means, the path length $\mathcal{H}^{1}(\gamma)$, and the length of the network $\mathcal{H}^{1}(\Sigma)$ :

$$
\Theta(a, b, c)=\bar{J}(a, b-a, c) .
$$

Obviously, $\Theta$ satisfies

$$
\Theta\left(\mathcal{H}^{1}(\gamma \backslash \Sigma), \mathcal{H}^{1}(\gamma), \mathcal{H}^{1}(\Sigma)\right)=\bar{J}\left(\mathcal{H}^{1}(\gamma \backslash \Sigma), \mathcal{H}^{1}(\gamma \cap \Sigma), \mathcal{H}^{1}(\Sigma)\right)
$$

We now study some properties of $\Theta$.

Proposition 4.4. $\Theta$ is monotone, non-decreasing with respect to each of its variables.

Proof. The monotonicity in the third variable is straightforward. The one in the first variable can be obtained observing that

$$
\Theta(a, b, c)=\inf _{a \leq s \leq b} J(s, b-s, c)
$$

and that the right-hand side of (4.5) is a non-decreasing function of $a$. The monotonicity in the second variable is obtained in a similar way, still relying on (4.5) and paying attention to the sets where the infimum is taken.

Proposition 4.5. $\Theta$ is lower semicontinuous.

Proof. We have to show that

$$
\Theta(a, b, c) \leq \liminf _{n \rightarrow+\infty} \Theta\left(a_{n}, b_{n}, c_{n}\right)
$$

when $a_{n} \rightarrow a, b_{n} \rightarrow b$ and $c_{n} \rightarrow c$. Let us consider for every real positive number $\varepsilon$ and for every positive integer $n$ a real number $s_{n}$ such that $a_{n} \leq s_{n} \leq b_{n}$ and

$$
J\left(s_{n}, b_{n}-s_{n}, c_{n}\right) \leq \Theta\left(a_{n}, b_{n}, c_{n}\right)+\varepsilon .
$$

Up to a subsequence, we can suppose that

$$
\liminf _{n \rightarrow+\infty} \Theta\left(a_{n}, b_{n}, c_{n}\right)=\lim _{n \rightarrow+\infty} \Theta\left(a_{n}, b_{n}, c_{n}\right) .
$$

We can also suppose that $s_{n} \rightarrow s$, where $a \leq s \leq b$. Thanks to the semicontinuity of $J$

$$
\Theta(a, b, c) \leq J(s, b-s, c) \leq \liminf _{n \rightarrow+\infty} J\left(s_{n}, b_{n}-s_{n}, c_{n}\right) \leq \liminf _{n \rightarrow+\infty} \Theta\left(a_{n}, b_{n}, c_{n}\right)+\varepsilon .
$$

Letting $\varepsilon \rightarrow 0^{+}$yields the desired inequality. 


\section{ExistenCE THEOREM}

In this section we continue to develop the tools we will use to prove Theorem 5.6.

Proposition 5.1. Let $\left\{x_{n}\right\}_{n \in \mathbb{N}}$ and $\left\{y_{n}\right\}_{n \in \mathbb{N}}$ be sequences in $K$ such that $x_{n} \rightarrow x$ and $y_{n} \rightarrow y$. If $\left\{\Sigma_{n}\right\}_{n \in \mathbb{N}}$ is a sequence of closed connected sets such that $\Sigma_{n} \rightarrow \Sigma$, then

$$
d_{\Sigma}(x, y) \leq \liminf _{n \rightarrow+\infty} d_{\Sigma_{n}}\left(x_{n}, y_{n}\right)
$$

Proof. First, up to a subsequence, we can suppose that

$$
\liminf _{n \rightarrow+\infty} d_{\Sigma_{n}}\left(x_{n}, y_{n}\right)=\lim _{n \rightarrow+\infty} d_{\Sigma_{n}}\left(x_{n}, y_{n}\right) .
$$

Given $\varepsilon>0$, we choose a sequence $\left\{\gamma_{n}\right\}_{n \in \mathbb{N}}$ such that $\gamma_{n} \in \mathscr{C}_{x_{n}, y_{n}}$ and

$$
\Theta\left(\mathcal{H}^{1}\left(\gamma_{n} \backslash \Sigma_{n}\right), \mathcal{H}^{1}\left(\gamma_{n}\right), \mathcal{H}^{1}\left(\Sigma_{n}\right)\right) \leq d_{\Sigma_{n}}\left(x_{n}, y_{n}\right)+\varepsilon
$$

Up to a subsequence we can suppose that $\gamma_{n} \rightarrow \gamma$ (it is easy to check that $x_{n} \rightarrow x$ and $y_{n} \rightarrow y$ imply $\gamma \in \mathscr{C}_{x, y}$ ) and

$$
\begin{aligned}
\mathcal{H}^{1}(\gamma \backslash \Sigma) & \leq \lim _{n} \mathcal{H}^{1}\left(\gamma_{n} \backslash \Sigma_{n}\right), \\
\mathcal{H}^{1}(\gamma) & \leq \lim _{n} \mathcal{H}^{1}\left(\gamma_{n}\right), \\
\mathcal{H}^{1}(\Sigma) & \leq \lim _{n} \mathcal{H}^{1}\left(\Sigma_{n}\right) .
\end{aligned}
$$

Using the semicontinuity and monotonicity of $\Theta$ (Props. 4.4 and 4.5), we obtain

$$
\begin{aligned}
d_{\Sigma}(x, y) & \leq \Theta\left(\mathcal{H}^{1}(\gamma \backslash \Sigma), \mathcal{H}^{1}(\gamma), \mathcal{H}^{1}(\Sigma)\right) \\
& \leq \Theta\left(\lim _{n \rightarrow+\infty} \mathcal{H}^{1}\left(\gamma_{n} \backslash \Sigma_{n}\right), \lim _{n \rightarrow+\infty} \mathcal{H}^{1}\left(\gamma_{n}\right), \lim _{n \rightarrow+\infty} \mathcal{H}^{1}\left(\Sigma_{n}\right)\right) \\
& \leq \liminf _{n \rightarrow+\infty} \Theta\left(\mathcal{H}^{1}\left(\gamma_{n} \backslash \Sigma_{n}\right), \mathcal{H}^{1}\left(\gamma_{n}\right), \mathcal{H}^{1}\left(\Sigma_{n}\right)\right) \\
& \leq \liminf _{n \rightarrow+\infty} d_{\Sigma_{n}}\left(x_{n}, y_{n}\right)+\varepsilon
\end{aligned}
$$

The arbitrary choice of $\varepsilon$ gives then inequality (5.6).

As a consequence of Proposition 5.1 we have the following corollary.

Corollary 5.2. Let $\left\{x_{n}\right\}_{n \in \mathbb{N}}$ and $\left\{y_{n}\right\}_{n \in \mathbb{N}}$ be sequences in $K$ such that $x_{n} \rightarrow x$ and $y_{n} \rightarrow y$. If $\Sigma$ is a closed connected set, then

$$
d_{\Sigma}(x, y) \leq \liminf _{n \rightarrow+\infty} d_{\Sigma}\left(x_{n}, y_{n}\right)
$$

In other words, $d_{\Sigma}$ is a lower semicontinuous function on $K \times K$.

Proposition 5.5 will play a crucial role in the proof of our main existence result. We split its proof in the next two lemmas for convenience.

Lemma 5.3. Let $X$ be a compact metric space, $\left\{f_{n}\right\}_{n \in \mathbb{N}}$ a sequence of positive real valued functions defined on $X$. Let also $g$ be a continuous positive real valued function defined on $X$. Then, the following statements are equivalent:

(1) $\forall \varepsilon>0 \exists N: \forall n \geq N \forall x \in X \quad g(x) \leq f_{n}(x)+\varepsilon$;

(2) $\forall x \in X \forall x_{n} \rightarrow x \quad g(x) \leq \liminf _{n} f_{n}\left(x_{n}\right)$. 
Proof.

- Let $x_{n} \rightarrow x$. Then

$$
g\left(x_{n}\right)=f_{n}\left(x_{n}\right)+\left(g\left(x_{n}\right)-f_{n}\left(x_{n}\right)\right) \leq f_{n}\left(x_{n}\right)+\varepsilon
$$

By the continuity of $g$, taking the lower limit we achieve

$$
g(x) \leq \liminf _{n \rightarrow+\infty} f_{n}\left(x_{n}\right)+\varepsilon
$$

Then $(1) \Rightarrow(2)$ is established when $\varepsilon \rightarrow 0^{+}$.

- Let us now prove that $(2) \Rightarrow(1)$. Suppose on the contrary that there exists a positive $\varepsilon$ and an increasing sequence of positive integers $\left\{n_{k}\right\}_{k}$ such that

$$
g\left(x_{n_{k}}\right) \geq f_{n_{k}}\left(x_{n_{k}}\right)+\varepsilon
$$

for a suitable $x_{n_{k}}$. Thanks to the compactness of $X$ we can suppose up to a subsequence that $x_{n_{k}} \rightarrow x$. Define

$$
x_{n}= \begin{cases}x_{n_{k}} & \text { if } n=n_{k} \text { for some } k \\ x & \text { otherwise }\end{cases}
$$

Then $x_{n} \rightarrow x$, and $g(x) \leq \liminf _{n} f_{n}\left(x_{n}\right)$. From (5.8) it follows,

$$
g(x) \geq \liminf _{k \rightarrow+\infty} f_{n_{k}}\left(x_{n_{k}}\right)+\varepsilon \geq \liminf _{n \rightarrow+\infty} f_{n}\left(x_{n}\right)+\varepsilon \geq g(x)+\varepsilon
$$

which is false.

Lemma 5.4. Let $f$ be a lower semicontinuous function defined on a metric space $(X, d)$ which ranges in $[0,+\infty]$. Then the set of functions $\left\{g_{t}: t \geq 0\right\}$ defined by

$$
g_{t}(x)=\inf \{f(y)+t d(x, y): y \in X\}
$$

satisfies the following properties:

- $g_{t} \geq 0$;

- $g_{t}$ is t-Lipschitz continuous;

- $g_{t}(x) \nearrow f(x)$ as $t \rightarrow+\infty$.

Proof. See Lemma 1.3.1 of [1] or Proposition 1.3.7 of [4].

Proposition 5.5. Let $\left\{f_{n}\right\}_{n \in \mathbb{N}}$ and $f$ be non-negative lower semicontinuous functions, all defined on a compact metric space $(X, d)$. Let $\left\{\mu_{n}\right\}_{n \in \mathbb{N}}$ be a sequence of nonnegative measures on $X$ such that $\mu_{n} \rightarrow^{*} \mu$. Suppose that

$$
\forall x \in X \forall x_{n} \rightarrow x \quad f(x) \leq \liminf _{n \rightarrow+\infty} f_{n}\left(x_{n}\right)
$$

Then

$$
\int_{X} f \mathrm{~d} \mu \leq \liminf _{n \rightarrow+\infty} \int_{X} f_{n} \mathrm{~d} \mu_{n} .
$$


Proof. Let $\psi$ be a continuous function with compact support such that $0 \leq \psi \leq 1$. Let $g_{t}$ be the function of Lemma 5.4; since $g_{t}$ satisfies the hypothesis of Lemma 5.3 with $g=g_{t}$, we have $g_{t} \leq f_{n}+\varepsilon$ for $n$ large enough and then

$$
\int_{X} g_{t} \psi \mathrm{d} \mu=\lim _{n \rightarrow+\infty} \int_{X} g_{t} \psi \mathrm{d} \mu_{n} \leq \liminf _{n \rightarrow+\infty} \int_{X} f_{n} \mathrm{~d} \mu_{n} .
$$

Taking the supremum in $t$ and $\psi$, we obtain

$$
\int_{X} f \mathrm{~d} \mu \leq \liminf _{n \rightarrow+\infty} \int_{X} f_{n} \mathrm{~d} \mu_{n} .
$$

We may now state and prove our existence result.

Theorem 5.6. The problem

$$
\min \{T(\Sigma): \Sigma \in \mathscr{C}\}
$$

admits a solution.

Proof. First, let us prove that for every $l>0$ the class

$$
\mathscr{D}_{l}:=\left\{\Sigma: \Sigma \in \mathscr{C}, \mathcal{H}^{1}(\Sigma) \leq l\right\}
$$

is a compact subset of the metric space $\left(\mathscr{C}(K), d_{\mathcal{H}}\right)$. Since $\left(\mathscr{C}(K), d_{\mathcal{H}}\right)$ is a compact space, it is enough to show that $\mathscr{D}_{l}$ is closed. We already know that the Hausdorff limit of a sequence of closed connected set is a closed connected set. If $\left\{\Sigma_{n}\right\}_{n \in \mathbb{N}}$ is a sequence of closed connected sets such that $\mathcal{H}^{1}\left(\Sigma_{n}\right) \leq l$

$$
\Sigma_{n} \rightarrow \Sigma \Longrightarrow \mathcal{H}^{1}(\Sigma) \leq \liminf _{n \rightarrow+\infty} \mathcal{H}^{1}\left(\Sigma_{n}\right) \leq l
$$

by Gołab theorem (Th. 3.2).

Second, by our assumption on the function $J$

$$
d_{\Sigma}(x, y) \geq G\left(\mathcal{H}^{1}(\Sigma)\right)
$$

so that

$$
T(\Sigma) \geq G\left(\mathcal{H}^{1}(\Sigma)\right)
$$

Then, if $\left\{\Sigma_{n}\right\}_{n \in \mathbb{N}}$ is a minimizing sequence, the sequence of 1-dimensional Hausdorff measures $\left\{\mathcal{H}^{1}\left(\Sigma_{n}\right)\right\}_{n \in \mathbb{N}}$ must be bounded, i.e. $\mathcal{H}^{1}\left(\Sigma_{n}\right) \leq l$, for some $l>0$.

If we prove that the functional $\Sigma \mapsto T(\Sigma)$ is sequentially lower semicontinuous on the class $\mathscr{D}_{l}$, then the existence of an optimal $\Sigma$ will be a consequence of the fact that a sequentially lower semicontinuous function takes a minimum on a compact metric space. Let $\left\{\Sigma_{n}\right\}_{n \in \mathbb{N}}$ be a sequence in $\mathscr{D}_{l}$ such that $\Sigma_{n} \rightarrow \Sigma$. Let $\left\{\mu_{n}\right\}_{n \in \mathbb{N}}$ be an optimal transport plan for the transport problem

$$
\min \left\{\int_{K \times K} d_{\Sigma_{n}}(x, y) \mathrm{d} \mu: \pi_{\#}^{+} \mu=\mu^{+}, \pi_{\#}^{-} \mu=\mu^{-}\right\} .
$$

Up to a subsequence we can suppose $\mu_{n} \rightarrow^{*} \mu$ for a suitable $\mu$. It is easy to see that $\mu$ is a transport plan between $\mu^{+}$and $\mu^{-}$.

Since by Proposition $5.1 d_{\Sigma}(x, y) \leq \liminf _{n} d_{\Sigma_{n}}\left(x_{n}, y_{n}\right)$ for all $x_{n} \rightarrow x$ and $y_{n} \rightarrow y$, by Lemma 5.5 we have

$$
\int_{K \times K} d_{\Sigma}(x, y) \mathrm{d} \mu \leq \liminf _{n \rightarrow+\infty} \int_{K \times K} d_{\Sigma_{n}}(x, y) \mathrm{d} \mu_{n} .
$$


Then by (5.9) we have

$$
T(\Sigma) \leq \int_{K \times K} d_{\Sigma}(x, y) \mathrm{d} \mu \leq \liminf _{n \rightarrow+\infty} \int_{K \times K} d_{\Sigma_{n}}(x, y) \mathrm{d} \mu_{n}=\liminf _{n \rightarrow+\infty} T\left(\Sigma_{n}\right) .
$$

We end with the following remark.

Remark 5.7. Note that if $\Sigma_{n}$ is a minimizing sequence, then the measure $\mu$ obtained in the proof of Theorem 5.6 is an optimal transport plan for the transport problem

$$
\min \left\{\int_{K \times K} d_{\Sigma}(x, y) \mathrm{d} \mu: \pi_{\#}^{+} \mu=\mu^{+}, \pi_{\#}^{-} \mu=\mu^{-}\right\} .
$$

Acknowledgements. This work is part of the European Research Training Network "Homogenization and Multiple Scales" (HMS2000) under contract HPRN-2000-00109. The authors also acknowledge the support of the project "Problemi di Ottimizzazione in Teoria del Trasporto ed Applicazioni a Problemi di Pianificazione Urbana" of the Italian GNAMPA and of the project "Calcolo delle Variazioni" of the Italian Ministry of Education.

\section{REFERENCES}

[1] L. Ambrosio and P. Tilli, Selected Topics on "Analysis on Metric Spaces". Appunti dei Corsi Tenuti da Docenti della Scuola, Scuola Normale Superiore, Pisa (2000).

[2] G. Bouchitté and G. Buttazzo, Characterization of Optimal Shapes and Masses through Monge-Kantorovich Equation. J. Eur. Math. Soc. (JEMS) 3 (2001) 139-168.

[3] A. Brancolini, Problemi di Ottimizzazione in Teoria del Trasporto e Applicazioni. Master's thesis, Università di Pisa, Pisa (2002). Available at http://www.sns.it/ brancoli/

[4] G. Buttazzo, Semicontinuity, Relaxation and Integral Representation in the Calculus of Variations. Pitman Research Notes in Mathematics Series 207. Longman Scientific \& Technical, Harlow (1989).

[5] G. Buttazzo and L. De Pascale, Optimal Shapes and Masses, and Optimal Transportation Problems, in Optimal Transportation and Applications (Martina Franca, 2001). Lecture Notes in Mathematics, CIME series 1813, Springer-Verlag, Berlin (2003) $11-52$.

[6] G. Buttazzo, E. Oudet and E. Stepanov, Optimal Transportation Problems with Free Dirichlet Regions, in Variational Methods for Discontinuous Structures (Cernobbio, 2001). Progress in Nonlinear Differential Equations and their Applications 51, Birkhäuser Verlag, Basel (2002) 41-65.

[7] G. Buttazzo and E. Stepanov, Optimal Transportation Networks as Free Dirichlet Regions for the Monge-Kantorovich Problem. Ann. Scuola Norm. Sup. Pisa Cl. Sci. 2 (2003) 631-678.

[8] G. Dal Maso and R. Toader, A Model for the Quasi-Static Growth of Brittle Fractures: Existence and Approximation Results. Arch. Rational Mech. Anal. 162 (2002) 101-135.

[9] K.J. Falconer, The Geometry of Fractal Sets. Cambridge Tracts in Mathematics, Cambridge University Press, Cambridge (1986).

[10] L.V. Kantorovich, On the Transfer of Masses. Dokl. Akad. Nauk. SSSR (1942).

[11] L.V. Kantorovich, On a Problem of Monge. Uspekhi Mat. Nauk. (1948).

[12] G. Monge, Mémoire sur la théorie des Déblais et des Remblais. Histoire de l'Acad. des Sciences de Paris (1781) 666-704.

[13] S.J.N. Mosconi and P. Tilli, $\Gamma$-convergence for the Irrigation Problem. Preprint Scuola Normale Superiore, Pisa (2003). Available at http://cvgmt.sns.it/ 